\title{
Buffered Electrochemical Polishing of Niobium
}

\author{
G.ianluigi Ciovati ${ }^{\mathrm{a}}$, Hui Tian ${ }^{\mathrm{a}, \mathrm{b}}$, Sean G. Corcoran ${ }^{\mathrm{c}}$,
}

${ }^{a}$ Thomas Jefferson National Accelerator Facility, Newport News, Virginia 23606, USA

${ }^{\mathrm{b}}$ Applied Science Department., College of William and Mary, Williamsburg, Virginia 23187, USA

'Materials Science \& Engineering Department., Virginia Tech, Blacksburg, Virginia 24061, USA

Correspondence to:

Gianluigi Ciovati

Thomas Jefferson National Accelerator Facility

12000 Jefferson Avenue

Newport News, VA 23606

Fax: 757-269-5945

e-mail: gciovati@jlab.org 


\begin{abstract}
The standard preparation of superconducting radio-frequency (SRF) cavities made of pure niobium include the removal of a "damaged” surface layer, by buffered chemical polishing (BCP) or electropolishing (EP), after the cavities are formed. The performance of the cavities is characterized by a sharp degradation of the quality factor when the surface magnetic field exceeds about $90 \mathrm{mT}$, a phenomenon referred to as “Q-drop”. In cavities made of polycrystalline fine grain (ASTM 5) niobium, the Q-drop can be significantly reduced by a low-temperature $\left(\sim 120^{\circ} \mathrm{C}\right)$ "in-situ” baking of the cavity if the chemical treatment was EP rather than BCP. As part of the effort to understand this phenomenon, we investigated the effect of introducing a polarization potential during buffered chemical polishing, creating a process which is between the standard BCP and EP. While preliminary results on the application of this process to $\mathrm{Nb}$ cavities have been previously reported, in this contribution we focus on the characterization of this novel electrochemical process by measuring polarization curves, etching rates, surface finish, electrochemical impedance and the effects of temperature and electrolyte composition. In particular, it is shown that the anodic potential of $\mathrm{Nb}$ during $\mathrm{BCP}$ reduces the etching rate and improves the surface finish.
\end{abstract}




\section{Introduction}

Niobium superconducting radio-frequency (SRF) cavities are widely used in modern particle accelerators, because of higher operating efficiency than normalconducting cavities made of copper. Since the superconducting RF current flows in a surface layer about $40 \mathrm{~nm}$ deep, a set of preparation techniques have been developed over the years to assure good superconducting properties of the surface layer [1]. In particular, the removal of a "damaged" layer of about $200 \mu \mathrm{m}$, resulting from the mechanical forming of the cavity, and a "contaminated" layer of about 20-50 $\mu \mathrm{m}$, resulting from the gettering of impurities by the $\mathrm{Nb}$ surface during high-temperature heat treatments, is achieved by either chemical etching or electropolishing. These chemical treatments result in a typical RMS surface roughness of about $1.6 \pm 0.42 \mu \mathrm{m}$ and about $0.34 \pm 0.11 \mu \mathrm{m}$, over a $50 \mu \mathrm{m} \times 50 \mu \mathrm{m}$ scan area, for buffered chemical polishing (BCP) and electropolishing (EP) respectively [2, 3]. The performance of SRF Nb cavities treated by BCP or EP is characterized by a sharp degradation of the quality factor above a surface magnetic field of about $90 \mathrm{mT}$; a phenomenon commonly referred to as “Q-drop”. It was found that a low-temperature baking $\left(\sim 120{ }^{\circ} \mathrm{C}, 12-48 \mathrm{~h}\right)$ of the cavities in ultra-high vacuum produced a significant improvement of the Q-drop in polycrystalline, fine-grain (ASTM 5) Nb cavities treated by EP but not BCP [4]. Therefore EP has emerged as the surface treatment of choice to produce cavities which can reach surface magnetic fields close to the theoretical limit of $\mathrm{Nb}$ [5]. However, the EP treatment is more expensive and time consuming than BCP. It should also be mentioned that recent research on cavities built from single-crystal and large-grain Nb and treated by BCP showed a smooth surface finish and performance comparable to electropolished cavities [6]. 
Several models have been proposed over the past ten years to explain the Q-drop and the baking effect but a comprehensive explanation of the experimental data is yet to be found. In particular, one model relates the Q-drop to a high density of localized states in the non-stochiometric niobium pentoxide $\mathrm{Nb}_{2} \mathrm{O}_{5-\mathrm{y}}$ (for example due to oxygen vacancies) which is readily formed on the niobium surface after exposing the pure metal to air or water [7]. Differences in the stochiometry and defect density between the oxide formed on an electropolished surface and the one formed on a chemically etched surface could account for the different cavity performance after baking.

During the standard BCP process, a mixture of hydrofluoric (49\%), nitric (70\%) and phosphoric acid (85\%) by volume ratio of 1:1:1 or 1:1:2 is used with a temperature range of $10-25{ }^{\circ} \mathrm{C}$. Niobium is oxidized by the nitric acid and the oxide layer is removed by the hydrofluoric acid, resulting in etching of the surface. This reaction is highly exothermic and the phosphoric acid is used as a buffer. During the standard electropolishing of niobium, anodic dissolution of the metal is obtained in a 1:9 volume ratio mixture of hydrofluoric (49\%) and sulfuric acid (96\%) at a temperature of $30-40{ }^{\circ} \mathrm{C}$ with a current density of $30-100 \mathrm{~mA} / \mathrm{cm}^{2}$.

The oxidation of niobium in the ambient atmosphere is a field assisted process (Cabrera-Mott model), where oxygen ions are attracted to the metal by a negative contact potential (Mott potential $\mathrm{V}_{\mathrm{M}} \sim-0.6 \mathrm{~V}$ ), yielding the $\mathrm{Nb}_{2} \mathrm{O}_{5}$ growth. In the early stage of growth, the oxide growth rate is limited by the diffusion of ions aided by the Mott potential, while in the later stage it is limited by the tunneling of electrons through the oxide aided by a positive ionic diffusion potential $\left(\mathrm{V}_{\mathrm{D}} \sim 0.17 \mathrm{~V}\right)$ limiting the $\mathrm{Nb}_{2} \mathrm{O}_{5}$ thickness to about $2-3 \mathrm{~nm}[8]$. 
In fluid electrolytes, the application of a small potential to the Nb cavity during BCP may enforce (anodic potential) or slow (cathodic potential) the oxidation process. Preliminary results on the performance of a Nb cavity after applying of this process are reported elsewhere [9] and no significant difference from the performance of a cavity treated by standard BCP was observed. In those experiments, a low voltage $(<1 \mathrm{~V}$, anodic or cathodic) and low current density (1-3 mA/ $\left.\mathrm{cm}^{2}\right)$ was applied between the cavity and a $\mathrm{Nb}$ rod coaxial to the cavity, used as counter electrode. The surface area ratio of the cavity to the Nb rod was 13:1.

In order to gain a better understanding of the electrochemical process occurring during the polarized BCP, we measured the anodic and cathodic polarization curves of $\mathrm{Nb}$ in the 1:1:2 and 1:1:1 mixtures, the resistivity of the electrolyte, the etching rates and surface finish for anodic and cathodic polarizations. These measurements have been done on both flat and cylindrical electrodes. In addition, electrochemical impedance spectroscopy (EIS) with rotating disk electrode (RDE) technique has been used for the 1:1:2 mixture to characterize the electrochemical process. If smoother surfaces can be obtained by applying a polarization to the cavity, this process may be included as a final step of a standard BCP treatment to improve the surface finish.

\section{Experimental}

In the flat electrode configuration, two high purity polycrystalline Nb $(99.9999 \%$, Teledyne Wah-Chang) samples with dimensions of $16 \mathrm{~mm}$ x $22 \mathrm{~mm} \times 3 \mathrm{~mm}$ are embedded into Teflon sample holders, providing an exposed surface area of $2.6 \mathrm{~cm}^{2}$ for the cathode and $1.4 \mathrm{~cm}^{2}$ for the anode. The separation between electrodes was $98 \mathrm{~mm}$. In the cylindrical electrode configuration, the anode consists of a high purity polycrystalline 
Nb cylinder (99.9999\%, Teledyne Wah-Chang) of $70 \mathrm{~mm}$ inner diameter, $3 \mathrm{~mm}$ thick, $100 \mathrm{~mm}$ height, sealed at one end with a Teflon disk. The cathode consists of a lower purity Nb tube (99.99\%, Teledyne Wah-Chang) of $29 \mathrm{~mm}$ outer diameter, $2 \mathrm{~mm}$ thick, $180 \mathrm{~mm}$ long, coaxial with the other electrode. The cylindrical electrodes configuration mimics the setup for polishing $\mathrm{Nb}$ cavities, while the flat electrodes configuration allows for easier analysis of the surface finish. Because most common metals will be etched by the BCP mixture, $\mathrm{Nb}$ was chosen as counter electrode in order to avoid possible contamination of the $\mathrm{Nb}$ working electrode by the counter electrode material; a situation which is unacceptable for the treatment of $\mathrm{Nb}$ cavities. The reference electrode was a saturated mercury mercurous-sulfate electrode (MSE) placed about $10 \mathrm{~mm}$ from the sample and about $5 \mathrm{~mm}$ from the cylinder, in the flat and cylindrical electrode configurations respectively. To protect the reference electrode from HF contamination, the electrode was placed in a standard reference bridge tube filled with $1 \mathrm{M} \mathrm{H}_{2} \mathrm{SO}_{4}$ as the conductive electrolyte. The electrolytes were prepared from reagent grade $49 \%$ hydrofluoric (48.8-49.2\%, J. T. Baker), 85\% phosphoric (85-87\%, J. T. Baker) and 70\% nitric acid (69-70\%, J. T. Baker).

In the flat electrode configuration, the Teflon block holding the samples is immersed in a large container filled with the electrolyte and the temperature is adjusted with a water bath. In the cylindrical electrodes configuration, the electrolyte is contained in the space between the two electrodes and the temperature is adjusted with a water bath. It is worthwhile to notice that in the cylindrical electrode configuration a much smaller volume of acid mixture is used for a much larger Nb surface, compared to the flat 
electrode configuration. The electrolyte was kept in static condition (no agitation) during all measurements.

A Sorensen DCS 33-33E power supply was used to apply a voltage, $\mathrm{V}_{\mathrm{ps}}$, between the Nb anode and cathode electrodes. The anode and cathode potentials were measured relative to the reference electrode, placed near the anode or cathode respectively, using a Keithley 6517A electrometer. For currents below 3 A, a HP 3478A multimeter was used, while the power supply readout was used for higher currents. The data acquisition was supported by a customized LabView program. The value of the current is the average of ten consecutive measurements.

Impedance measurements were performed with a Gamry Instruments G300 potentiostat controlled with a commercial software package (Gamry Instrument Framework). Impedance measurements were performed at a constant applied dc potential added to a small ac potential (10 $\mathrm{mV}$ rms amplitude) in the frequency range $200 \mathrm{kHz}$ to $0.2 \mathrm{~Hz}$. A Gamry RDE710 was used for the rotating electrode setup. For EIS measurements, a high purity polycrystalline $\mathrm{Nb}$ disc electrode (19.63 $\mathrm{mm}^{2}$ surface area) was inserted in a Teflon holder. The $\mathrm{Nb}$ disc was mechanically polished up to 4000 grit emery paper, cleaned in isopropanol with ultrasonic agitation and rinsed with distilled water and air dried. A high purity $\mathrm{Al}$ wire was used as the counter electrode.

\section{Results}

Polarization behavior - Figures 1 and 2 show the typical polarization behavior for the anode and cathode in the flat and cylindrical electrode configurations, respectively. The open circuit potential of the $\mathrm{Nb}$ in the $\mathrm{HF}: \mathrm{HNO}_{3}: \mathrm{H}_{3} \mathrm{PO}_{4}=1: 1: 2$ mixture at $2{ }^{\circ} \mathrm{C}$, relative to the reference electrode is about $-0.150 \pm 0.005 \mathrm{~V}(+0.490 \mathrm{~V}$ vs. SHE). This potential is 
measured during active dissolution of the metal by the acid mixture and represents the potential of $\mathrm{Nb}$ ions in the niobium pentoxide. Current oscillations occurred for anodic potential of $\mathrm{Nb}$ in the range $0.5-1.7 \mathrm{~V}$ vs. MSE followed by a current “plateau” at higher anodic potential. The amplitude of the current oscillations was larger in the cylindrical electrodes configuration than in the flat electrodes one: the peak current was about 30\% higher than the average value in the former case, while it was only about $10 \%$ higher than the average value in the latter. This may be due to a higher local temperature of the anode in the cylindrical than in the flat sample configuration.

The polarization curve was also measured with the $\mathrm{HF}: \mathrm{HNO}_{3}: \mathrm{H}_{3} \mathrm{PO}_{4}=1: 1: 1$ mixture for the cylindrical electrode configuration. Larger current densities were obtained at the same anodic potentials, as shown in Fig. 2. The polarization curve was limited to below $1 \mathrm{~V}$ vs. MSE as the large current produced large heating and bubbling in the electrolyte.

The resistivity of the electrolyte was approximated from the difference in anode potential measured relative to two different reference electrode locations (one near the anode and one near the cathode), as a function of the current flow, for current density up to $\sim 100 \mathrm{~mA} / \mathrm{cm}^{2}$. The value obtained for both flat and cylindrical electrodes configurations was about $35 \mathrm{~m} \Omega \mathrm{cm}$ at bath temperatures of $10-23{ }^{\circ} \mathrm{C}$. Figure 3 shows a high sensitivity of the anodic limiting current density as a function of the electrolyte temperature, obtained from the polarization data. This may result in non-uniform polishing effects when local temperature conditions are not well controlled.

Surface topography - Images of the flat anode and cathode surfaces after processing were taken with a Hirox KH-3000VD digital optical microscope. In the first experiment, the 
electrodes were polished in the current oscillation region $\left(\mathrm{V}_{\mathrm{ps}}=1.7 \mathrm{~V}, \mathrm{~V}_{\text {anode }}=0.966 \mathrm{~V}\right.$ vs. MSE, $\mathrm{J}=83 \mathrm{~mA} / \mathrm{cm}^{2}, \mathrm{~T}_{\text {bath }}=24{ }^{\circ} \mathrm{C}$ ) for about $1 \mathrm{~h}$, using the 1:1:2 mixture as the electrolyte. Analysis of the surface finish by optical microscopy, profilometry and atomic force microscopy of both the anode and cathode showed grain boundary etching and roughness comparable to what is typically achieved with the standard buffered chemical polishing. In the second experiment, the electrodes were polished in the current plateau region $\left(\mathrm{V}_{\mathrm{ps}}=3 \mathrm{~V}, \mathrm{~V}_{\text {anode }}=2.2 \mathrm{~V}\right.$ vs. MSE, $\left.\mathrm{J}=200 \mathrm{~mA} / \mathrm{cm}^{2}, \mathrm{~T}_{\text {bath }}=10^{\circ} \mathrm{C}\right)$ for about $1 \mathrm{~h}$, using the 1:1:2 mixture as the electrolyte. Optical microscopy of the anode (Fig. 4a) shows a smooth surface, somewhat similar to electropolishing, while the cathode finish resembles that of standard BCP (Fig. 4b). Nevertheless, some regions of the anode showed several pits (Fig. 4c). The density of pits, their average radius and depth were 131 pits/ $\mathrm{cm}^{2}, 40 \pm 6 \mu \mathrm{m}$ (average of 34 pits) and $21 \pm 7 \mu \mathrm{m}$ (average of 15 pits), respectively. A high purity $\mathrm{Nb}$ sample was etched in the same 1:1:2 mixture, without any potential applied, and the surface finish is shown in Fig. 4d for comparison. No pits were observed on either the cathode or on the buffered chemical polished sample. The material removal was measured by weight loss and with a micrometer and the resulting etching rates were $1.15 \mu \mathrm{m} / \mathrm{min}, 0.67 \mu \mathrm{m} / \mathrm{min}$ and $1.03 \mu \mathrm{m} / \mathrm{min}$ for the cathode, anode and chemically polished Nb samples, respectively. The RMS value of the surface roughness of the anode was measured over an area $50 \mu \mathrm{m} \times 50 \mu \mathrm{m}$ with an atomic force microscope (Digital Instrument Dimension Nanoscope IV operated in the Tapping Mode with an ultrasharp silicon tip of a diameter of $10 \mathrm{~nm}$ ). The average value of the RMS roughness for eight different locations on the anode is $113 \pm 41 \mathrm{~nm}$, comparable to values measured on electropolished surfaces. The average value of the peak-to-valley height is $759 \pm 151 \mathrm{~nm}$. 
Figure 5 shows a plot of the surface topography over a $50 \mu \mathrm{m} \times 50 \mu \mathrm{m}$ area measured by atomic force microscopy (AFM).

Impedance experiments - Impedance measurements were performed using a freshly prepared $\mathrm{HF}: \mathrm{HNO}_{3}: \mathrm{H}_{3} \mathrm{PO}_{4}$ mixture (1:1:2 by volume) as a function of applied potential, with no agitation, and at a fixed potential (4 V relative to MSE) but different speed of the rotating disk electrode. During all measurements, the temperature of the bulk electrolyte was maintained at $14{ }^{\circ} \mathrm{C}$. Nyquist plots of the electrochemical impedance as a function of frequency for several values of the applied dc potential and for several angular velocities are shown in Figs. 6 and 7, respectively. From Fig. 6, three loops covering the frequency ranges of $1.6-5 \mathrm{~Hz}, 5-5000 \mathrm{~Hz}$ and $5-200 \mathrm{kHz}$ were noticed. The diameter of the high-frequency loop increases with increasing applied potential while it decreases with increasing rotation speed. In the low-frequency range, the magnitude of the impedance increases as the potential is increased. The data taken with the rotating disk electrode shown in Fig. 7 have larger scatter then those taken in static condition, most likely due to the large turbulence near the electrode because of the low-viscosity acid mixture used in these experiments. This may also explain why the data became extremely noisy at rotation speeds above $100 \mathrm{rpm}$. A plot of the reciprocal of the limiting current density as a function of the reciprocal of the square root of the angular velocity is shown in Fig. 8. The data follow a straight line with non-zero intercept, indicating that the current plateau in the polarization curve is not due to mass-transport limitation only but by a mixed mass-transport and kinetic control. The results from the EIS experiments will be discussed more in details in the next section.

\section{Discussion}


The polarization curve of $\mathrm{Nb}$ in the same electrolyte used for buffered chemical polishing shows similarities with the polarization curve in $\mathrm{HF}: \mathrm{H}_{2} \mathrm{SO}_{4}=1: 9$ electrolyte, used for the electropolishing of $\mathrm{Nb}$ : current oscillations occur within a certain range of the applied anodic potential, followed by a plateau region at higher voltages. The plateau region is commonly associated with a mechanism of limited mass transport of species such as the electrolyte anions and the cations and water molecules across a diffusion layer between $\mathrm{Nb}$ and the bulk solution. A recent investigation of electropolishing on $\mathrm{Nb}$ revealed the presence of a compact salt film and that the diffusion-limited access of $\mathrm{F}^{-}$ anion to the film limits the local reaction rate, causing the limited-current plateau [10]. The electrolyte used for EP of $\mathrm{Nb}$ has a mass fraction of sulfuric acid of about $90 \%$. Sulfuric acid has a high viscosity and low dissociation constant $\left(\mathrm{pK}_{\mathrm{a}}=-3\right)$, so that the oxidation reaction occurring at the anode can be written as [11]:

$$
2 \mathrm{Nb}+5 \mathrm{SO}_{4}{ }^{2-}+5 \mathrm{H}_{2} \mathrm{O} \rightarrow \mathrm{Nb}_{2} \mathrm{O}_{5}+10 \mathrm{H}^{+}+5 \mathrm{SO}_{4}{ }^{2-}+10 \mathrm{e}^{-}
$$

For comparison, the mass fraction of hydrofluoric, nitric and phosphoric acids and water used for $\mathrm{BCP}$ is given in Table $\mathrm{I}$. $\mathrm{HNO}_{3}(70 \%)$ has the lowest dissociation constant among the acids used in the BCP mixture $\left(\mathrm{pK}_{\mathrm{a}}=-1.4\right)$, so that the oxidation of $\mathrm{Nb}$ at the anode could be enforced by both $\mathrm{NO}_{3}{ }^{-}$anions and water:

$$
\begin{aligned}
& 8 \mathrm{Nb}+10 \mathrm{NO}_{3}{ }^{-} \rightarrow 4 \mathrm{Nb}_{2} \mathrm{O}_{5}+10 \mathrm{NO}(\mathrm{g})+10 \mathrm{e}^{-} \\
& 2 \mathrm{Nb}+10 \mathrm{NO}_{3}{ }^{-}+5 \mathrm{H}_{2} \mathrm{O} \rightarrow \mathrm{Nb}_{2} \mathrm{O}_{5}+10 \mathrm{H}^{+}+10 \mathrm{NO}_{3}{ }^{-}+10 \mathrm{e}^{-} .
\end{aligned}
$$

In all processes, $\mathrm{Nb}_{2} \mathrm{O}_{5}$ is then dissolved by $\mathrm{HF}$ as soluble niobium fluoride or oxyfluoride. The phosphoric acid would have the same "buffer" role as during standard BCP. Although micro-smoothening of the Nb surface resulted from the application of the anodic potential and a smoother surface than by standard BCP was achieved, the data 
from the rotating disk electrode shown in Fig. 7 and the presence of pits, shown in Fig. 4c, suggest that mixed mass-transport and kinetic control mechanism occurs in the current plateau region of the polarization curve. Pitting is also often observed on electropolished $\mathrm{Nb}$ samples and cavities and it has been related to a degradation of cavity performance [12], most likely because of magnetic field enhancement at the edges of the pits, and should therefore be avoided. The stronger dependence of current density on temperature in the $\mathrm{HF}: \mathrm{HNO}_{3}: \mathrm{H}_{3} \mathrm{PO}_{4}=1: 1: 2$ electrolyte than in $\mathrm{HF}: \mathrm{H}_{2} \mathrm{SO}_{4}=1: 9$, may make it more favorable for the formation of etching pits. In addition, hydrogen bubbles formed on the anode surface from the process in Eq. [3] may also be responsible for pitting and potentially hydrogen loading of the niobium. Some degree of electrolyte agitation near the electrode, which is a standard practice during BCP etching but which was not applied in these experiments, and a good temperature control, for example by flowing water on the back side of the electrode, should help reducing the probability of pitting on the surface.

The etching rate and surface finish of the cathode do not differ significantly from that of standard BCP, so the reaction most likely to occur there, besides BCP etching, is just the reduction of hydronium ions into hydrogen gas or nitrogen oxide:

$$
\begin{aligned}
& 2 \mathrm{H}^{+}+2 \mathrm{e}^{-} \rightarrow \mathrm{H}_{2}(\mathrm{~g}) \\
& 3 \mathrm{H}^{+}+3 \mathrm{e}^{-}+\mathrm{HNO}_{3} \rightarrow \mathrm{NO}+2 \mathrm{H}_{2} \mathrm{O}
\end{aligned}
$$

More information about the mechanisms governing the plateau region of the polarization curve can be obtained from the analysis of the EIS data. The presence of multiple loops in the Nyquist plot had been previously observed for the electropolishing of Fe13Cr in a phosphoric acid-based electrolyte [13]. In that particular system the high- 
frequency loop is associated with a charge-transfer process and the lowest frequency loop is associated with the diffusion of a solvating acceptor species. The intermediate frequency loop is related to the formation of adsorbed ionic species which compete for available surface sites. The current plateau and the electropolishing of Fe13Cr resulted from the transport limitation of the acceptor species from the bulk electrolyte to the surface of the dissolving metal [14].

The high-frequency loop of the Nyquist plot in the current plateau region for $\mathrm{Nb}$ in $\mathrm{HF}: \mathrm{HNO}_{3}: \mathrm{H}_{3} \mathrm{PO}_{4}=1: 1: 2$ electrolyte has the shape of a "depressed" semicircle. It is described with an equivalent circuit, shown in Fig. 9, which includes the solution resistance, $R_{s}$, the polarization resistance, $R_{p}$, and a constant phase element of impedance $Z=1 /(j \omega)^{\alpha} Y_{0} . Y_{0}$ corresponds to the double-layer capacitance, $C_{d l}$, for $\alpha=1$. The values of the parameters of the equivalent circuit have been obtained from a fit of the highfrequency loop data. The results for different values of the polarization potential and rotation speed are shown in Tables II and III, respectively. The solution resistance is nearly independent of rotation speed while it seems to decrease with increasing potential. This last feature might not be accurate since the impedance at the highest frequency value (200 kHz) increases significantly with increasing potential, introducing larger errors in the calculation of the fit parameter $\mathrm{R}_{\mathrm{s}}=\mathrm{Z}(\infty)$. The polarization resistance increases with increasing potential while the effective double-layer capacitance decreases. These behaviors are consistent with the presence of a compact salt film on the metal surface [10]. On the other hand, the dependence of the model parameters on rotation speed favors the adsorbates-acceptor model: $R_{s}, C_{d l}$ as well as the product $i_{s s} R_{p}$ do not change with rotation speed [13]. Among the factors which complicate the interpretation of the EIS 
data, there is the presence of a standard chemical etching superimposed to the electrochemical polishing and the much lower viscosity of the electrolyte used for this study, compared to typical electrolytes used for EP. An estimate of the kinematic viscosity using Refutas equation gives $1.9 \mathrm{~mm}^{2} / \mathrm{s}$ for $\mathrm{HF}$ (49\%): $\mathrm{HNO}_{3}(70 \%): \mathrm{H}_{3} \mathrm{PO}_{4}$ $(85 \%)=1: 1: 2$, compared to about $9.2 \mathrm{~mm}^{2} / \mathrm{s}$ for the $\mathrm{HF}(49 \%): \mathrm{H}_{2} \mathrm{SO}_{4}(96 \%)=1: 9$ electrolyte used for the electropolishing of $\mathrm{Nb}$. It is therefore possible that agitation of the electrolyte may disrupt a thin compact film on the $\mathrm{Nb}$ anode and favor a multi-step polishing mechanism involving adsorbed intermediates, as proposed in the adsorbatesacceptor model.

Even though, in the current plateau region, the anodic polarization of $\mathrm{Nb}$ in the BCP mixture causes the polishing rate to be reduced by about a factor of two than in the standard BCP, some chemical etching, producing large amounts of niobium fluoride or oxyfluoride, may still occur, due to the large quantities of $\mathrm{HF}$ and $\mathrm{HNO}_{3}$ present in the electrolyte. One of the factors which most likely favor a diffusion-controlled reaction at the anode should be the local concentration of $\mathrm{F}^{-}$, which may be reduced from the value in the bulk electrolyte by the presence of a compact film on the surface. This would be consistent with the higher etching rate obtained in this electrolyte than in HF $(49 \%): \mathrm{H}_{2} \mathrm{SO}_{4}(96 \%)=1: 9$ electrolyte and with the observation that much higher current density is obtained for the same applied voltage, if the relative HF concentration is increased, as for the $\mathrm{HF}: \mathrm{HNO}_{3}: \mathrm{H}_{3} \mathrm{PO}_{4}=1: 1: 1$ mixture (as shown in the polarization curve in Fig. 2). Another important factor in establishing a diffusion-controlled reaction could be the transport limitation of the acceptor species: as large amounts of reactions products are formed on the anode surface, the ability of solvating species, such as water, 
to reach the surface could limit the reaction rate. Clearly, further studies will be necessary to better identify the limiting mechanism and, in particular, a wider range of water concentrations and temperature values should be explored.

\section{Conclusion}

Buffered electrochemical polishing of niobium in a mixture of phosphoric, nitric and hydrofluoric acid has been explored for application to the surface preparation of $\mathrm{Nb}$ microwave resonators for particle accelerators. Applying a polarizing potential to $\mathrm{Nb}$ immersed in the same acid mixture commonly used for buffered chemical polishing was explored as an attempt to alter the oxide layer on the $\mathrm{Nb}$ surface and evaluate its influence on high-field RF losses. The electrochemical process was studied by measuring polarization curves and electrochemical impedance as a function of frequency, with and without electrolyte agitation. In the current plateau region, micro-polishing occurred, yielding smoother surfaces than by standard BCP process. As Nb cavities are typically subjected to several hundred microns of material removal by BCP after fabrication, a smoother surface could therefore be achieved by applying an anodic potential to the cavity, filled with the same electrolyte, as a final polishing step. The removal rate of the polarized BCP is about a factor of two lower than standard BCP but about a factor of three faster than standard electropolishing of $\mathrm{Nb}$. Further optimization of process parameters such as temperature and acid flow rate is necessary, particularly to minimize pitting. For the experimental conditions examined in this study, the presence of mixed kinetic and mass-transport control makes the identification of the transport limiting species, responsible for microsmoothing, particularly challenging. We propose fluorine ions or water-related species as the most likely candidates. 


\section{Acknowledgements}

The authors would like to acknowledge O. Trofimova of the College of William and Mary for helping with the optical microscope and AFM measurements.

This research was conducted at Thomas Jefferson National Accelerator Facility, operated by Jefferson Science Associates, LLC under U.S. DOE Contract No. DE-AC0506OR23177. The U.S. Government retains a non-exclusive, paid-up, irrevocable, worldwide license to publish or reproduce this manuscript for U.S. Government purposes. 


\section{References}

1. P. Kneisel, Nucl. Inst. \& Methods A, 557, 250 (2006).

2. L.Lilje, Ph. D. Thesis, University of Hamburg, 2001.

3. H. Tian, Ph. D. Thesis, College of William \& Mary, 2008.

4. G. Ciovati, Physica C 441, 44 (2006).

5. K. Saito, in Proceedings of the 13th SRF Workshop, Beijing, China, TU202 (2007).

6. P. Kneisel, G. R. Myneni, G. Ciovati, J. Sekutowicz, and T. Carneiro, in Proceedings of the Single Crystal-Large Grain Niobium Technology: International Niobium Workshop, Araxa, Brasil, AIP Conference Proceedings, Vol. 927 (2007), p. 84.

7. J. Halbritter, P. Kneisel, V. Palmieri, and M. Pekeler, IEEE Trans. Appl. Supercond. 11, 1864 (2001).

8. J. Halbritter, Appl. Phys. A 43, 1 (1987).

9. G. Ciovati, S. G. Corcoran, and J. Halbritter, in Proceedings of the 13th SRF Workshop, Beijing, China, TUP17 (2007).

10. H. Tian, S. G. Corcoran, C. E. Reece and M. J. Kelley, J. Electrochem. Soc. 155, D563 (2008).

11. P. Kneisel, in Proceedings of the 1st SRF Workshop, Karlsruhe, Germany, (1980), p. 27.

12. Y. Iwashita, Y Tajima, and H. Hayano, Phys. Rev. ST Accel. Beams 11, 093501 (2008).

13. S. Magaino, M. Matlosz, and D. Landolt, J. Electrochem. Soc. 140, 1365 (1993).

14. M. Matlosz, S. Magaino, and D. Landolt, J. Electrochem. Soc. 141, 410 (1994). 
Figure 1. Typical polarization behavior for flat $\mathrm{Nb}$ electrodes in $\mathrm{HF}(49 \%): \mathrm{HNO}_{3}$ (70\%): $\mathrm{H}_{3} \mathrm{PO}_{4}(85 \%)=1: 1: 2$ (volume ratio) at $20{ }^{\circ} \mathrm{C}$. The surface area ratio of the anode to cathode was 1.94:1. The voltage between the anode and cathode was controlled in a two electrode configuration. The potential of the electrodes were measured using a saturated mercury mercurous sulfate reference electrode (MSE).

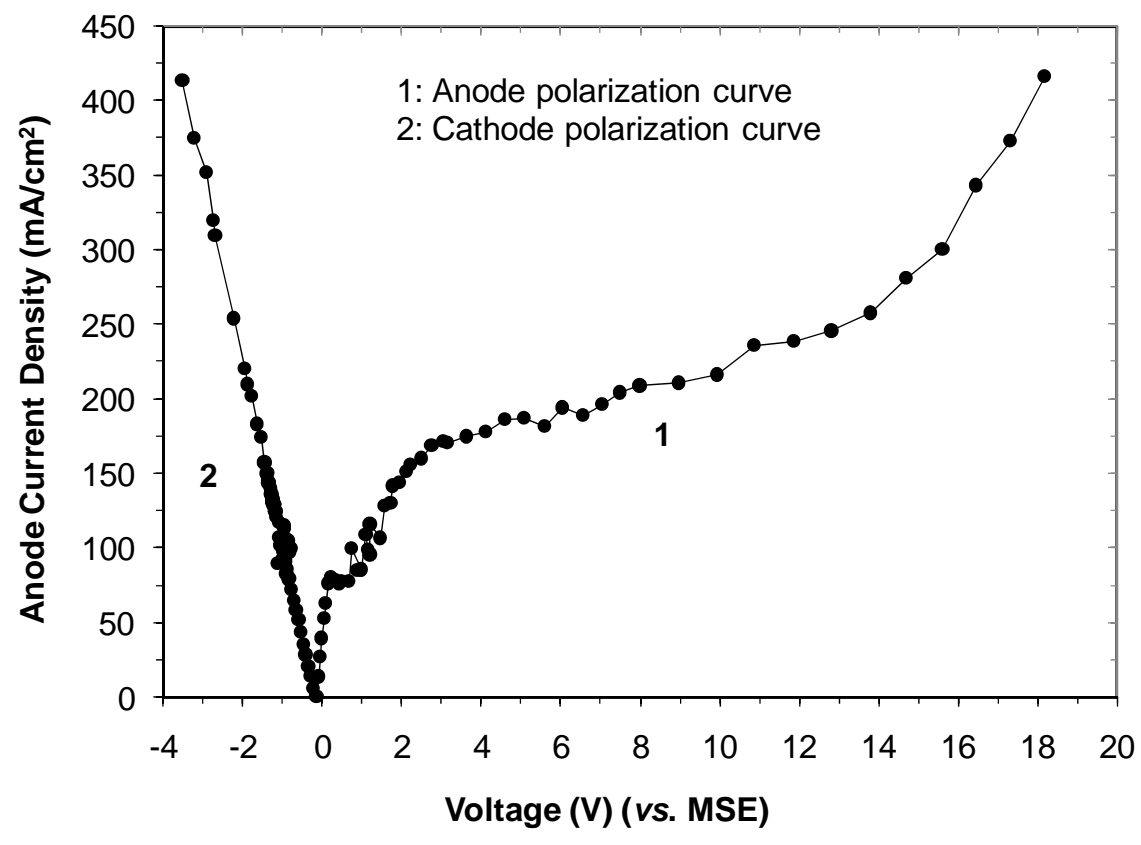


Figure 2. Typical polarization behavior for cylindrical Nb electrodes in $\mathrm{HF}(49 \%): \mathrm{HNO}_{3}$ $(70 \%): \mathrm{H}_{3} \mathrm{PO}_{4}(85 \%)=1: 1: 2$ (solid circles) and 1:1:1 (empty triangles) by volume ratio at $15{ }^{\circ} \mathrm{C}$ and $25^{\circ} \mathrm{C}$, respectively. The surface area ratio of anode to cathode was 2.3:1. The voltage between the anode and cathode is controlled in a two electrode configuration. The potential of the electrodes was measured using a saturated mercury mercurous sulfate reference electrode (MSE).

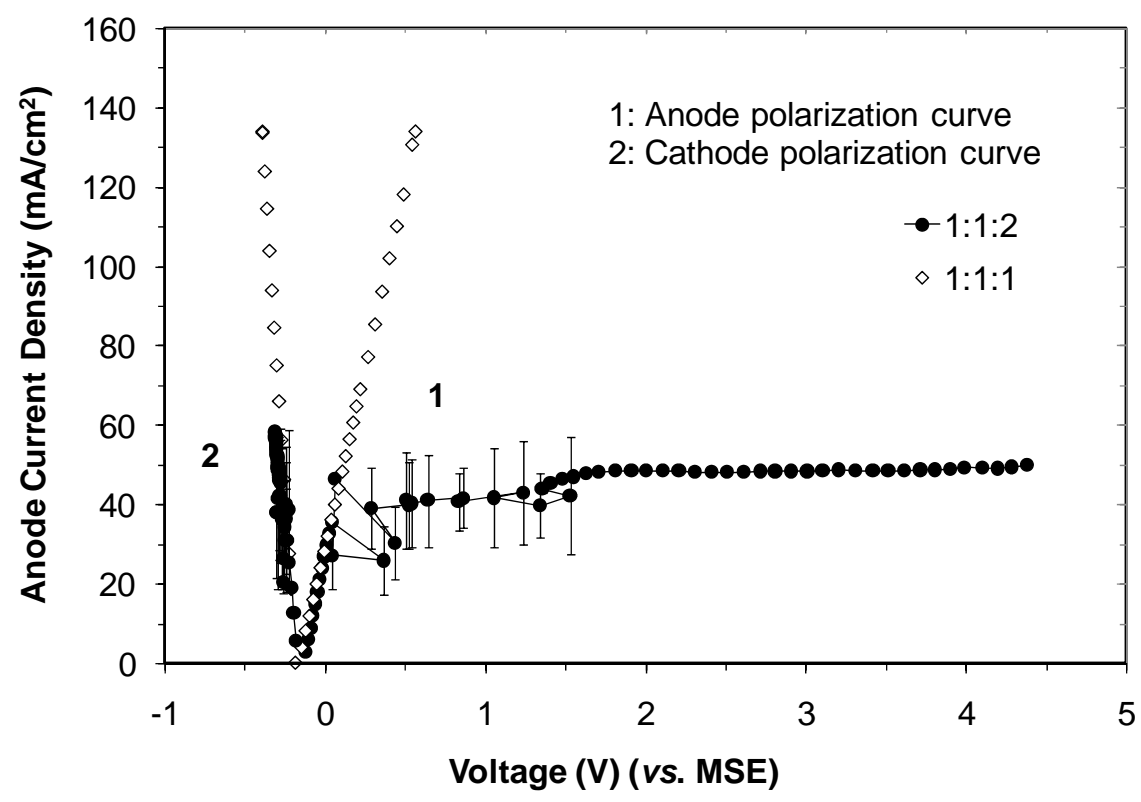


Figure 3. Anodic limiting current density as a function of the temperature of the electrolyte ( $\mathrm{HF}(49 \%): \mathrm{HNO}_{3}(70 \%): \mathrm{H}_{3} \mathrm{PO}_{4}(85 \%)$ = 1:1:2 by volume).

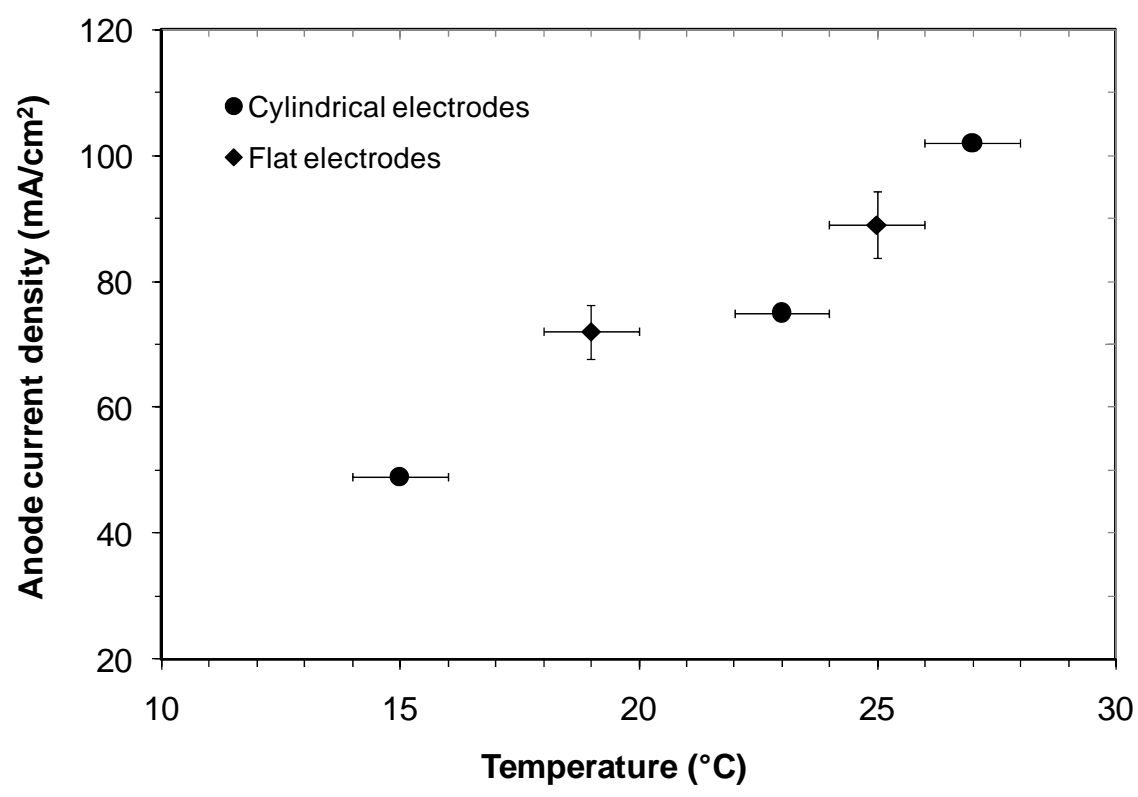


Figure 4. Surface finish of flat $\mathrm{Nb}$ anode (a) and cathode (b) after polishing in the plateau region at $\mathrm{J}=200 \mathrm{~mA} / \mathrm{cm}^{2}, \mathrm{~T}_{\text {bath }}=10{ }^{\circ} \mathrm{C}$ in the $\mathrm{HF}(49 \%): \mathrm{HNO}_{3}(70 \%): \mathrm{H}_{3} \mathrm{PO}_{4}(85 \%)=$ 1:1:2 electrolyte. Pits were observed in some regions of the anode (c), while the surface finish of a Nb sample etched in the same solution is shown in (d). The size of each picture corresponds to a surface area of about $770 \mu \mathrm{m} \times 590 \mu \mathrm{m}$.
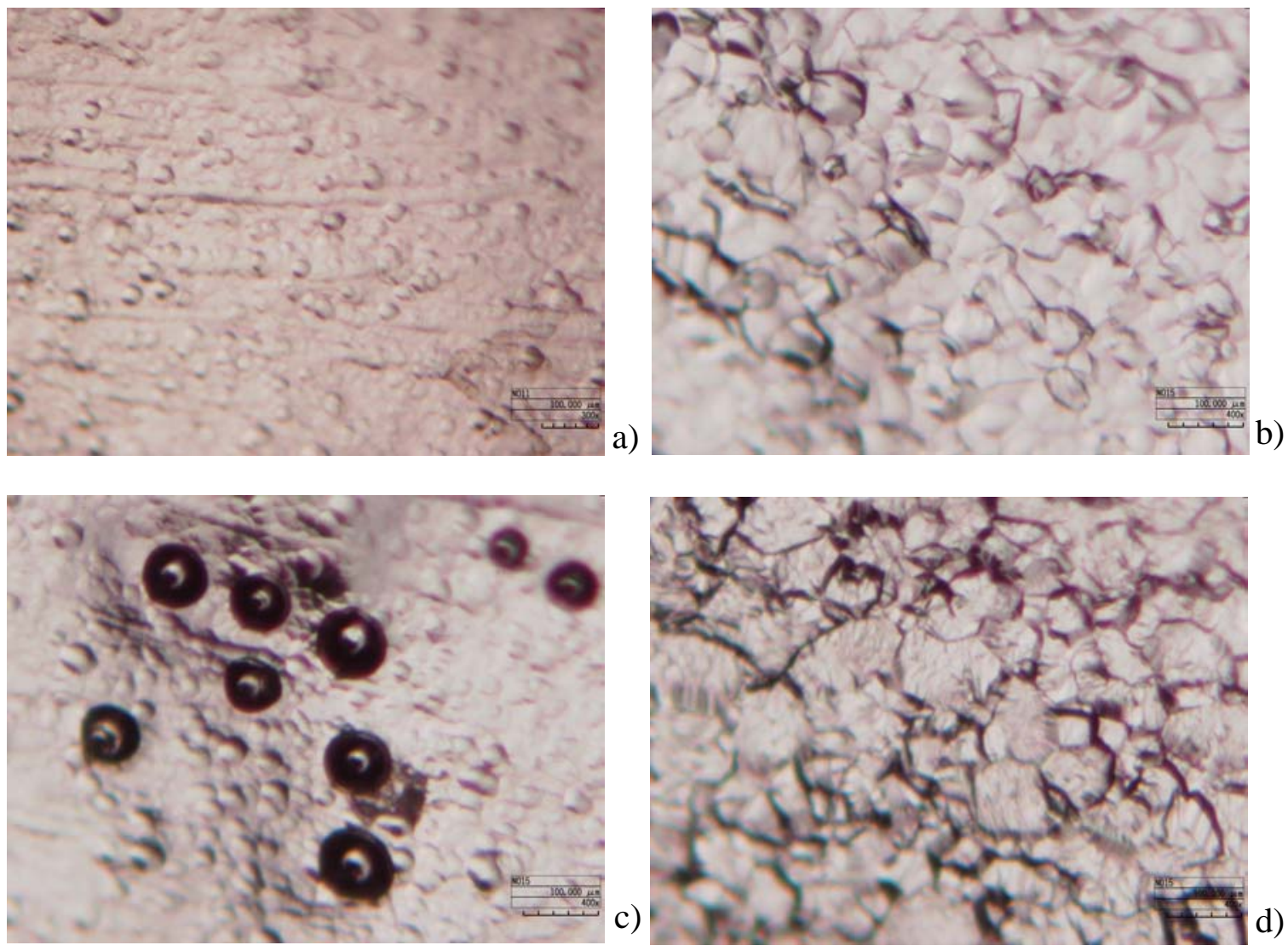
Figure 5. Surface topography measured by AFM on a $50 \mu \mathrm{m} \times 50 \mu \mathrm{m}$ area of the $\mathrm{Nb}$ anode polished in the plateau region at $\mathrm{J}=200 \mathrm{~mA} / \mathrm{cm}^{2}, \mathrm{~T}_{\text {bath }}=10{ }^{\circ} \mathrm{C}$ in the $\mathrm{HF}$ (49\%): $\mathrm{HNO}_{3}(70 \%): \mathrm{H}_{3} \mathrm{PO}_{4}(85 \%)=1: 1: 2$ electrolyte. The RMS roughness is $105 \mathrm{~nm}$.

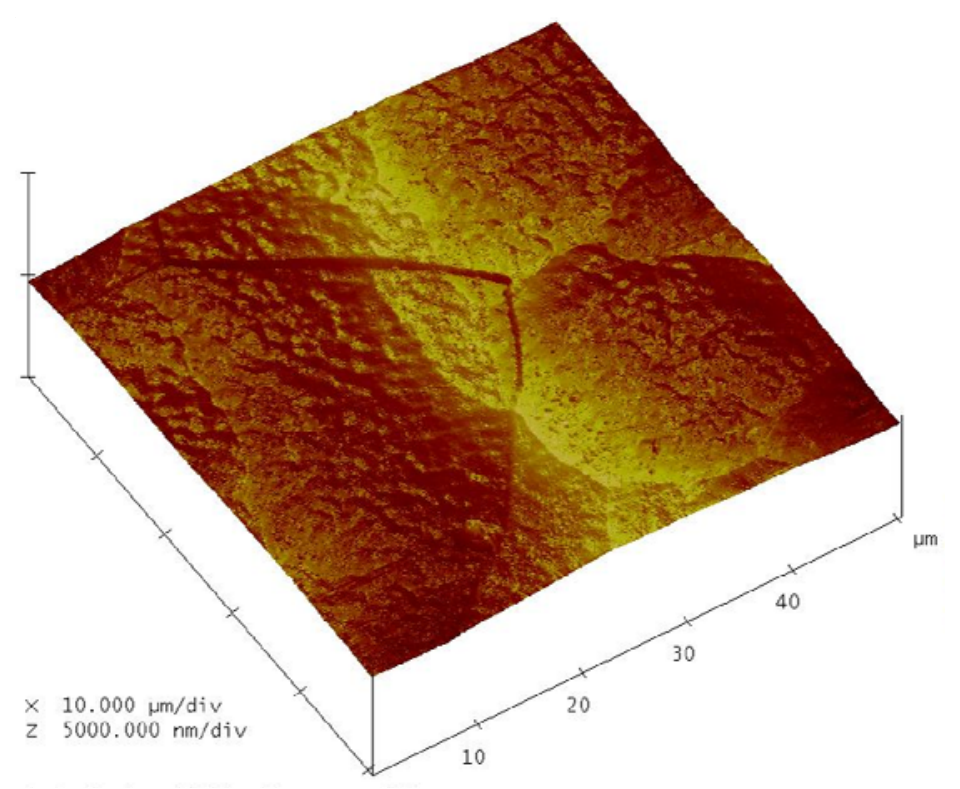


Figure 6. Impedance diagrams for $\mathrm{Nb}$ in $\mathrm{HF}(49 \%): \mathrm{HNO}_{3}(70 \%): \mathrm{H}_{3} \mathrm{PO}_{4}(85 \%)=1: 1: 2$ electrolyte at $14{ }^{\circ} \mathrm{C}$ measured at different applied potentials in the current plateau region.

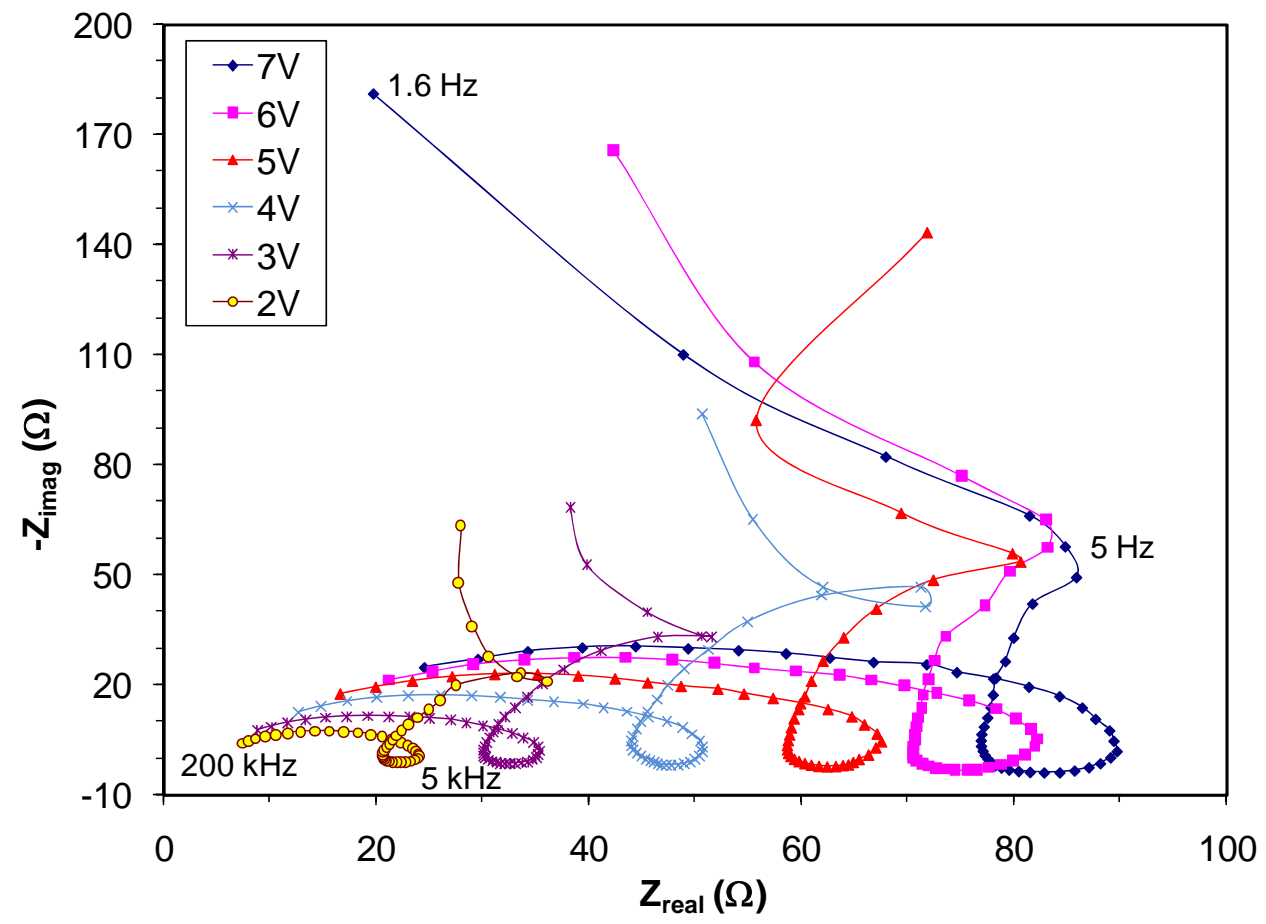


Figure 7. Effect of rotation speed on the impedance diagram for $\mathrm{Nb}$ in $\mathrm{HF}(49 \%): \mathrm{HNO}_{3}$ (70\%): $\mathrm{H}_{3} \mathrm{PO}_{4}(85 \%)=1: 1: 2$ electrolyte at $14{ }^{\circ} \mathrm{C}$ and applied potential of $4 \mathrm{~V}$ vs. MSE.

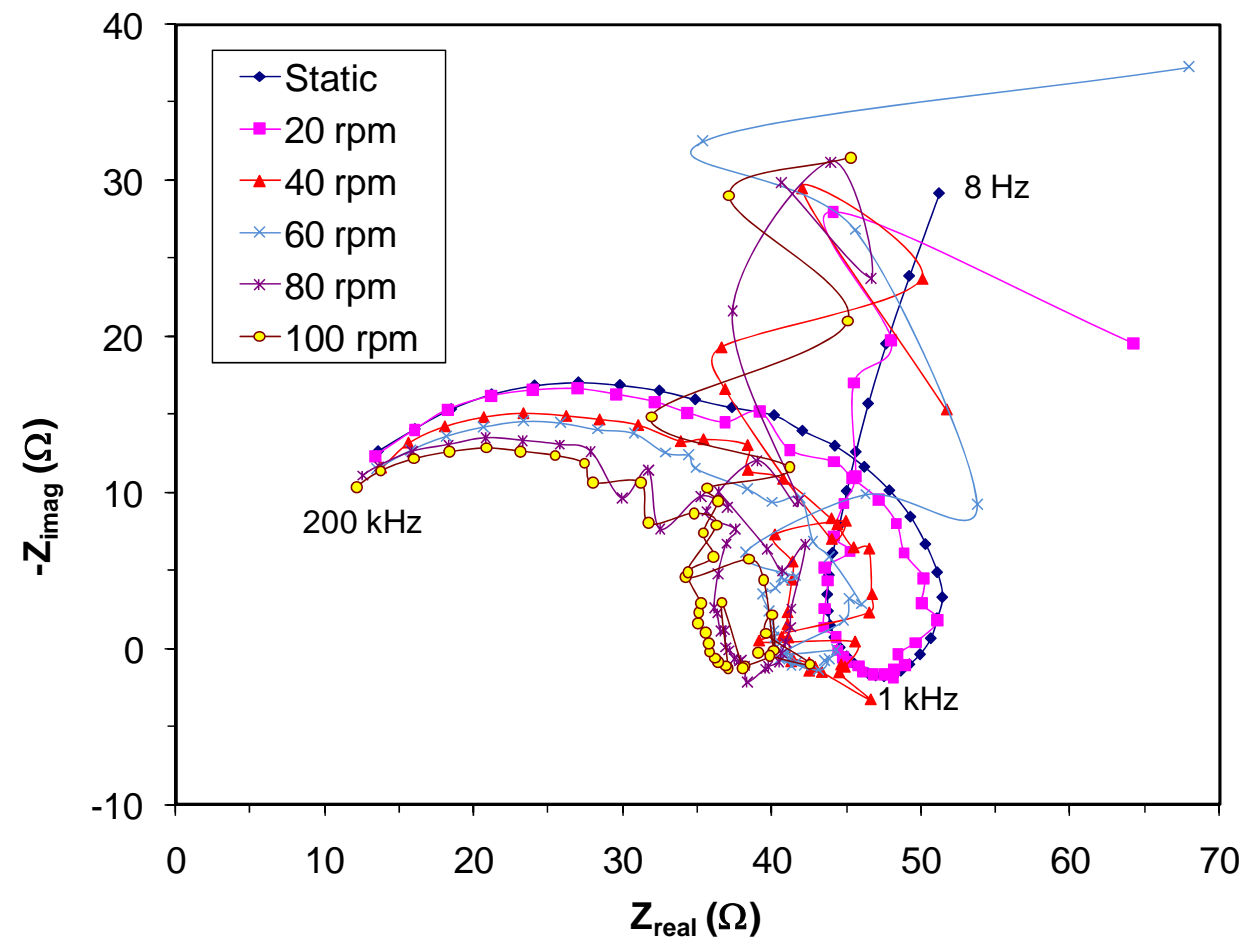


Figure 8. Relation between the reciprocal of the limiting current density and the reciprocal of the square root of angular rotation speed determined for $\mathrm{Nb}$ in $\mathrm{HF}$ (49\%): $\mathrm{HNO}_{3}(70 \%): \mathrm{H}_{3} \mathrm{PO}_{4}(85 \%)=1: 1: 2$ at $14{ }^{\circ} \mathrm{C}$. The solid line is a linear fit of the data.

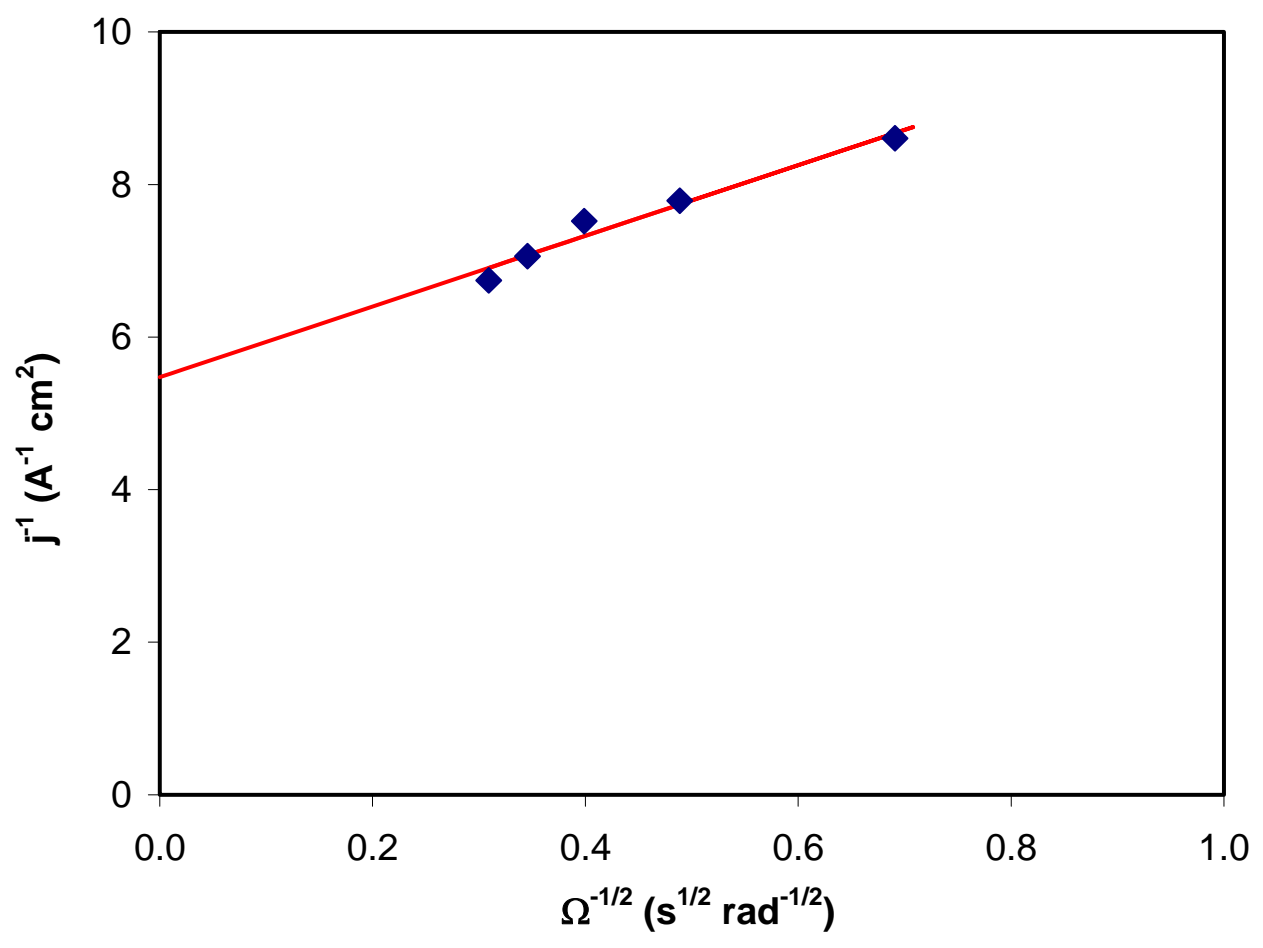


Figure 9. Equivalent circuit used to describe the high-frequency part of the impedance diagrams.

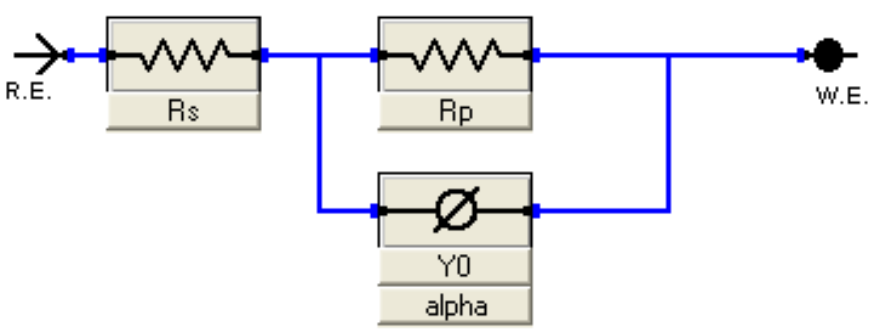


Table I. Mass fractions of the components of the acid mixtures used for BCP and EP.

\begin{tabular}{|c|c|c|c|c|}
\hline & $\begin{array}{c}\text { Reagent Conc. } \\
\text { (Mass \%) }\end{array}$ & BCP 1:1:1 & BCP 1:1:2 & EP \\
\hline $\mathrm{HF}$ & 49 & 0.134 & 0.096 & 0.033 \\
\hline $\mathrm{HNO}_{3}$ & 70 & 0.233 & 0.167 & - \\
\hline $\mathrm{H}_{3} \mathrm{PO}_{4}$ & 85 & 0.337 & 0.484 & - \\
\hline $\mathrm{H}_{2} \mathrm{SO}_{4}$ & 96 & - & - & 0.896 \\
\hline $\mathrm{H}_{2} \mathrm{O}$ & 100 & 0.295 & 0.253 & 0.071 \\
\hline
\end{tabular}


Table II. Variation of $\mathrm{R}_{\mathrm{s}}, \mathrm{R}_{\mathrm{p}}$ and $\mathrm{C}_{\mathrm{dl}}$ with potential for $\mathrm{Nb}$ in $\mathrm{HF}$ (49\%):HNO (70\%): $\mathrm{H}_{3} \mathrm{PO}_{4}(85 \%)=1: 1: 2$ at $14{ }^{\circ} \mathrm{C}$ and static electrolyte condition.

\begin{tabular}{|c|c|c|c|}
\hline $\begin{array}{c}\text { Potential } \\
(V \text { vs. MSE })\end{array}$ & $\begin{array}{c}\mathrm{R}_{\mathrm{s}} \\
\left(\Omega \mathrm{cm}^{2}\right)\end{array}$ & $\begin{array}{c}\mathrm{R}_{\mathrm{p}} \\
\left(\Omega \mathrm{cm}^{2}\right)\end{array}$ & $\begin{array}{c}\mathrm{C}_{\mathrm{dl}} \\
\left(\mu \mathrm{F} / \mathrm{cm}^{2}\right)\end{array}$ \\
\hline 2 & 0.91 & 4.10 & 1.07 \\
\hline 3 & 0.51 & 6.89 & 0.51 \\
\hline 4 & 0.18 & 10.74 & 0.26 \\
\hline 5 & 0.06 & 13.91 & 0.19 \\
\hline 6 & 0.02 & 17.10 & 0.15 \\
\hline 7 & 0.02 & 18.86 & 0.13 \\
\hline
\end{tabular}


Table III. Variation of $\mathrm{R}_{\mathrm{s}}, \mathrm{R}_{\mathrm{p}}$ and $\mathrm{C}_{\mathrm{dl}}$ with rotation speed for $\mathrm{Nb}$ in $\mathrm{HF}$ (49\%):HNO $(70 \%): \mathrm{H}_{3} \mathrm{PO}_{4}(85 \%)=1: 1: 2$ at $14{ }^{\circ} \mathrm{C}$ and $4 \mathrm{~V}$ vs. MSE. Also included is the product of the steady-state current, $\mathrm{i}_{\mathrm{ss}}$, and the polarization resistance.

\begin{tabular}{|c|c|c|c|c|}
\hline $\begin{array}{c}\text { Rotation speed } \\
(\mathrm{rpm})\end{array}$ & $\begin{array}{c}\mathrm{R}_{\mathrm{s}} \\
\left(\Omega \mathrm{cm}^{2}\right)\end{array}$ & $\begin{array}{c}\mathrm{R}_{\mathrm{p}} \\
\left(\Omega \mathrm{cm}^{2}\right)\end{array}$ & $\begin{array}{c}\mathrm{C}_{\mathrm{dl}} \\
\left(\mu \mathrm{F} / \mathrm{cm}^{2}\right)\end{array}$ & $\begin{array}{c}\mathrm{i}_{\mathrm{ss}} \mathrm{R}_{\mathrm{p}} \\
(\mathrm{V})\end{array}$ \\
\hline 20 & 0.33 & 10.34 & 0.26 & 1.20 \\
\hline 40 & 0.36 & 9.49 & 0.27 & 1.22 \\
\hline 60 & 0.39 & 8.98 & 0.26 & 1.19 \\
\hline 80 & 0.32 & 8.58 & 0.26 & 1.22 \\
\hline 100 & 0.32 & 7.67 & 0.26 & 1.14 \\
\hline
\end{tabular}

